\title{
Therapeutic Drug Monitoring and Nephrotoxicity of Teicoplanin Therapy in Chinese Children: A Retrospective Study
}

This article was published in the following Dove Press journal: Infection and Drug Resistance

\author{
Dan Sun ${ }^{1, *}$ \\ Tao Zhang ${ }^{1} *$ \\ Jie $\mathrm{Mi}^{2}$ \\ Yuzhu Dong ${ }^{3}$ \\ Yang Liu' \\ Ying Zhang' \\ Di Zhang' \\ Taotao Wang' \\ Hua Cheng ${ }^{2}$ \\ Yalin Dong' \\ 'Department of Pharmacy, The First \\ Affiliated Hospital of Xi'an Jiaotong \\ University, Xi'an, Shaanxi 71006I, \\ People's Republic of China; ${ }^{2}$ Department \\ of Pharmacy, Xi'an Children's Hospital, \\ Xi'an, Shaanxi 710003, People's Republic \\ of China; ${ }^{3}$ Department of Pharmacy, The \\ Third Affiliated Hospital of Chongqing \\ Medical University, Chongqing 40II 20, \\ People's Republic of China
}

*These authors contributed equally to this work
Purpose: This study aims to 1) describe the distribution characteristics of teicoplanin trough concentration $\left(C_{\min }\right)$ and explore the related influencing factors and 2) evaluate the nephrotoxicity of teicoplanin in children.

Patients and Methods: A cohort of children who were treated with teicoplanin intravenously were included in this retrospective study. Regression analysis was performed to explore the factors associated with the fluctuations of teicoplanin $C_{\min }$ and the development of nephrotoxicity. Classification and regression tree analysis was used to identify the population at high risk for teicoplanin nephrotoxicity.

Results: A total of 269 plasma samples from 186 children were collected. Underexposure $\left(C_{\min }<10 \mathrm{mg} / \mathrm{L}\right)$ was documented in $52.7 \%$ of cases. The $C_{\min } /$ dose after administering the loading dose was strongly associated with age $(P=0.008)$, weight $(P=0.039)$, and serum creatinine $(P=0.022)$. The $C_{\min } /$ dose after administering the maintenance dose was strongly associated with gender $(P=0.014)$ and serum creatinine $(P=0.006) . C_{\min }(P=0.012)$ and the concomitant treatment with amphotericin B $(P=0.001)$ were the independent risk factors for teicoplanin-related nephrotoxicity. Children who were concomitantly treated by amphotericin B with teicoplanin $C_{\min }>9.81 \mathrm{mg} / \mathrm{L}$ or patients with teicoplanin $C_{\min }>21.94 \mathrm{mg} / \mathrm{L}$ were at high risk for nephrotoxicity.

Conclusion: The fluctuations of teicoplanin $C_{\min }$ could be affected by age, weight, gender, and serum creatinine. $C_{\min }$ and concomitant treatment with amphotericin B were the independent risk factors for nephrotoxicity. We suggested that the therapeutic drug monitoring of teicoplanin should be performed in children.

Keywords: teicoplanin, trough concentration, children, nephrotoxicity, factor

\section{Introduction}

Gram-positive bacterial infections remain a significant health concern in pediatric patients. ${ }^{1,2}$ Teicoplanin is a glycopeptide antibiotic exhibiting high antibacterial activities against the majority of gram-positive bacteria. It is widely used for the treatment of skin and soft tissue infections, bone and joint infections, pneumonia, urinary tract infections and infective endocarditis in pediatrics worldwide, ${ }^{3}$ as the superior tissue penetration, slower rate of elimination and fewer adverse reactions compared with vancomycin. ${ }^{4}$

Previous studies showed that teicoplanin trough concentration $\left(C_{\min }\right)$ was significantly associated with improved clinical response, and a $C_{\min }$ of $>60 \mathrm{mg} / \mathrm{L}$ would increase the likelihood of nephrotoxicity..$^{5-7}$ Generally, it is recommended
Department of Pharmacy, The First

Affiliated Hospital of Xi'an Jiaotong

University, Xi'an, Shaanxi 71006I,

People's Republic of China

Tel/Fax +86-29-8532324l

Email dongyalin@mail.xjtu.edu.cn

Hua Cheng

Department of Pharmacy, Xi'an Children's Hospital, Xi'an, Shaanxi 7I0003, People's

Republic of China

$\mathrm{Tel} / \mathrm{Fax}+86-29-87692090$

Email chhyjk@I63.com
Infection and Drug Resistance 2020: I3 4|05-4II3

4105

DovePress if in $b$

http://doi.org/10.214711DR.S272982 
that, for most gram-positive infections, teicoplanin $C_{\min }$ should be maintained at least $10 \mathrm{mg} / \mathrm{L}$ (measured by high performance liquid chromatography, HPLC) or $15 \mathrm{mg} / \mathrm{L}$ (measured by fluorescence polarization immunoassay, FPIA) in clinical practice. ${ }^{8,9}$ In addition, large interindividual and intra-individual variation of teicoplanin $C_{\text {min }}$ have been observed in both adult and pediatric patients, ${ }^{10-16}$ and it has been reported that drug underexposure was documented in more than half of the cases. ${ }^{3}$ Weight, serum creatinine and other clinical factors were found to be significantly correlated with the $C_{\min }$ of teicoplanin. ${ }^{3,17-19}$

Clinical data focused on therapeutic drug monitoring (TDM) of teicoplanin in pediatric patients, especially in children, is very limited. And factors influencing teicopla$\operatorname{nin} C_{\min }$ have not been fully elucidated. Yamada et al evaluated the $C_{\min }$ of teicoplanin after the recommended loading dose in 36 children and found that age and renal function were significantly associated with teicoplanin $C_{\text {min. }}{ }^{17}$ Another large-scale retrospective TDM study (280 pediatric patients) conducted by Strenger et al indicated that age and gender contributed to the variation of teicoplanin concentration. ${ }^{19}$ However, more researches are needed to further explore the influencing factors of teicoplanin $C_{\min }$ due to the limited data.

Besides, compared to vancomycin, teicoplanin lead to a lower risk of nephrotoxicity. ${ }^{20,21}$ However, nephrotoxicity is associated with greater hospital costs and longer length of stay in non-critically ill children. ${ }^{22}$ To date, only Yamada et al reported that the incidence of nephrotoxicity in children was $2.3 \%$, and no significant difference in renal impairment was found between the groups with $C_{\min }$ $>20 \mathrm{mg} / \mathrm{L}$ and $C_{\text {min }}<20 \mathrm{mg} / \mathrm{L}$ (measured by FPIA). ${ }^{17}$ However, the risk factors of teicoplanin-related nephrotoxicity have not been fully explored in children.

In this regard, this study aimed to conduct a retrospective study to 1$)$ describe the distribution characteristics of teicoplanin $C_{\min }$ and explore the related influencing factors associated with $C_{\min } ; 2$ ) analyze and evaluate the incidence of teicoplanin-related nephrotoxicity and its risk factors in children.

\section{Patients and Methods Study Population}

In this retrospective study, we included a cohort of children who were treated with teicoplanin intravenously at the First Affiliated Hospital of Xi'an Jiaotong University and Xi'an Children's Hospital between March 2017 and December 2018. Inclusion criteria were: 1) patients aged from 2 months to 18 years old; 2) patients who were clinically diagnosed or suspected with gram-positive bacterial infections, such as respiratory infections, bloodstream infections, bone and joint infections, skin and soft tissue infections and so on, and then were treated with teicoplanin (for children aged 2 months to 12 years, loading dose: one single dose of $10 \mathrm{mg} / \mathrm{kg}$ body weight administered intravenously every 12 hours, repeated 3 times; maintenance dose: one single dose of $6-10 \mathrm{mg} / \mathrm{kg}$ body weight administered intravenously once a day. For children aged $>12$ years, loading dose: $6 \mathrm{mg} / \mathrm{kg}$ body weight every 12 hours for 3 intravenous or intramuscular administrations; maintenance dose: $6 \mathrm{mg} / \mathrm{kg}$ body weight intravenous or intramuscular once a day). The specific prescription of each patient was determined by physicians; 3) patients who underwent TDM of teicoplanin. Exclusion criteria were: 1) patients who underwent continuous renal replacement therapy or peritoneal dialysis; 2) patients who received teicoplanin for less than 48 hours. This study protocol was approved by the Ethics Committee of the two hospitals.

\section{Data Collection}

The patient's clinical data were extracted through the hospital's electronic medical record system and the nursing system. The main information was as follows: 1) demographic data, such as gender, age, and weight; 2) underlying diseases and diagnosis of infection; 3) dosage regimen of teicoplanin and the concomitant medication (defined as the other drugs were given at or almost at the same day with teicoplanin before performing TDM for more than one day); 4) laboratory test results, such as creatinine, transaminase (aspartate aminotransferase and alanine transaminase) and platelet count; 5) adverse reactions information, such as nephrotoxicity.

\section{Blood Sample Collection and Concentration Measurement}

At least 3 days after the first dose of teicoplanin, plasma samples were taken from patients 30 minutes before the next dose. A validated HPLC method established by our research group was used to quantify the plasma concentrations of teicoplanin. The linear range was $5.63-125.00 \mathrm{mg} / \mathrm{L}$ $(r=0.9995)$. The minimum limit of quantitation was $5.63 \mathrm{mg} / \mathrm{L}$. The average relative standard deviations of 
intra-day and inter-day were $3.2 \%$ and $6.8 \%$, respectively, and the mean relative error of intra-day and inter-day were $0.12 \%$ and $0.10 \%$, respectively. ${ }^{23}$

\section{Definitions}

Teicoplanin $C_{\min }$ were divided into two groups: $C_{\min }$ in the first group were the plasma concentrations measured on day 3 or 4 after the first dose of teicoplanin. $C_{\min }$ in another group were the plasma concentrations measured at steady state (the teicoplanin dosage was not changed for $\geq 72 \mathrm{~h}$ ). ${ }^{24}$ Underexposure was defined as the $C_{\min }$ of teicoplanin was less than $10 \mathrm{mg} / \mathrm{L}$.

To explore factors affecting the fluctuation of teicoplanin $C_{\text {min }}$, we performed an analysis to investigate the relationship between different factors and the value of $C_{\text {min }} /$ Dose (C/D). C/D were divided into two groups: one was $C^{3 / 4}{ }_{\min } / \mathrm{D}_{\mathrm{CLD}}$ (the ratio between $C_{\min }$ on day 3 or day 4 after teicoplanin administration and the cumulative loading dose), and the other was $C^{\mathrm{SS}}{ }_{\min } / \mathrm{D}_{\mathrm{MD}}$ (the ratio between the teicoplanin $C_{\min }$ at steady state and the maintenance dose). Nephrotoxicity was defined as a serum creatinine $(\mathrm{SCr})$ increase at least 1.5 times that of the baseline. $^{25}$

\section{Statistical Analysis}

Statistical analyses were performed using SPSS 19.0. The mean \pm standard deviation (SD) or median (interquartile range, IQR) was used to describe continuous variables depending on the normality of distribution, whereas percent or count was used to describe categorical variables. Univariate and multivariate linear regression analysis was applied to determine potential factors affecting the teicopla$\operatorname{nin} C / \mathrm{D}$. Univariate and multivariate logistic regression analyses were applied to explore the potential risk factors for nephrotoxicity. Variables with $P<0.1$ in the univariate analysis were combined in the multivariate analysis. Logistic regression and classification and regression tree (CART) analysis were performed to identify the patient population with a greater or less likelihood of developing nephrotoxicity. In this study, variables with $P<0.1$ in a multivariate logistic regression analysis were included in the CART analysis.

\section{Results}

\section{Baseline Patient Characteristics}

One hundred and eighty-six children were included in this study. Patients were typically male $(56.5 \%)$, with a median age of 3.82 years (IQR 1.53-6.27 years). The median weight was $15.3 \mathrm{~kg}$ (IQR 11.0-21.0 kg). The main reason for teicoplanin therapy was respiratory infections $(58.1 \%)$ and malignancy was the most common comorbid condition (46.2\%). Only $25.8 \%$ of patients were proven to be infected with gram-positive bacterial. Median albumin and $\mathrm{SCr}$ were $35.0 \mathrm{~g} / \mathrm{L}$ and $26 \mu \mathrm{mol} / \mathrm{L}$, respectively (Table 1 ).

\section{Distribution of Teicoplanin Trough Concentrations}

The distribution of teicoplanin $C_{\min }$ was shown in Figure 1. A total of 269 teicoplanin $C_{\min }$ were included in this study. The median of $C_{\min }$ was $9.49 \mathrm{mg} / \mathrm{L}$ (IQR 5.97-15.35 mg/L)

Table I Patients' Characteristics

\begin{tabular}{|l|l|}
\hline Characteristics & Value \\
\hline Number of patients, $\mathrm{n}$ & 186 \\
Gender, males/female, $\mathrm{n}(\%)$ & $105 / 8 \mathrm{I}(56.5 \% / 43.5 \%)$ \\
Age (year), median (IQR) & $3.82(1.53,6.27)$ \\
Weight (kg), median (IQR) & $15.3(11.0,21.0)$ \\
Teicoplanin loading dose (mg/kg), median (IQR) & $10.0(9.7,10.00)$ \\
Teicoplanin maintenance dose (mg/kg), median & $10.00(9.7,10.00)$ \\
(IQR) & \\
Duration of teicoplanin therapy (days), median & $12(9,16)$ \\
(IQR) & \\
\hline Type of infection, $\mathrm{n}$ (\%) & \\
Skin and soft tissue infection & $14(7.5 \%)$ \\
Respiratory infection & $108(58.1 \%)$ \\
Bloodstream infection & $45(24.2 \%)$ \\
Bone and joint infections & $17(9.1 \%)$ \\
Other infections & $2(1.1 \%)$ \\
\hline Isolated gram-positive organisms, $\mathrm{n}(\%)$ & \\
Staphylococcus & $18(9.7 \%)$ \\
Enterococcus & $9(4.8 \%)$ \\
Streptococcus & $9(4.8 \%)$ \\
Others & $12(6.5 \%)$ \\
\hline Comorbid conditions, $\mathrm{n}(\%)$ & \\
Malignancy & $86(46.2 \%)$ \\
Congenital heart disease & $21(11.3 \%)$ \\
Hypoalbuminemia & $32(17.2 \%)$ \\
Baseline WBC (I0\%/L), median (IQR) & $5.24(1.20,11.23)$ \\
Baseline NEUT\%, median (IQR) & $52.1(28.9,69.7)$ \\
Baseline albumin (g/L), median (IQR) & $35.0(30.7,39.6)$ \\
Baseline ALT (U/L), median (IQR) & $20.0(12.0,42.0)$ \\
Baseline AST (U/L), median (IQR) & $27.5(20.0,43.0)$ \\
Baseline SCr ( $\mu$ mol/L), median (IQR) & $95.2(66.9,127.2)$ \\
Baseline Ccr (mL/min) ${ }^{a}$, median (IQR) & \\
\hline Note: Est & \\
\hline
\end{tabular}

Note: ${ }^{a}$ Estimated by Cockcroft-Gault.

Abbreviations: IQR, interquartile range; $C_{\min }$, trough concentration; WBC, white blood cell; NEUT\%, neutrophil percentage; ALT, alanine aminotransferase; AST, aspartate aminotransferase; $\mathrm{SCr}$, serum creatinine; $\mathrm{Ccr}$, creatinine clearance. 

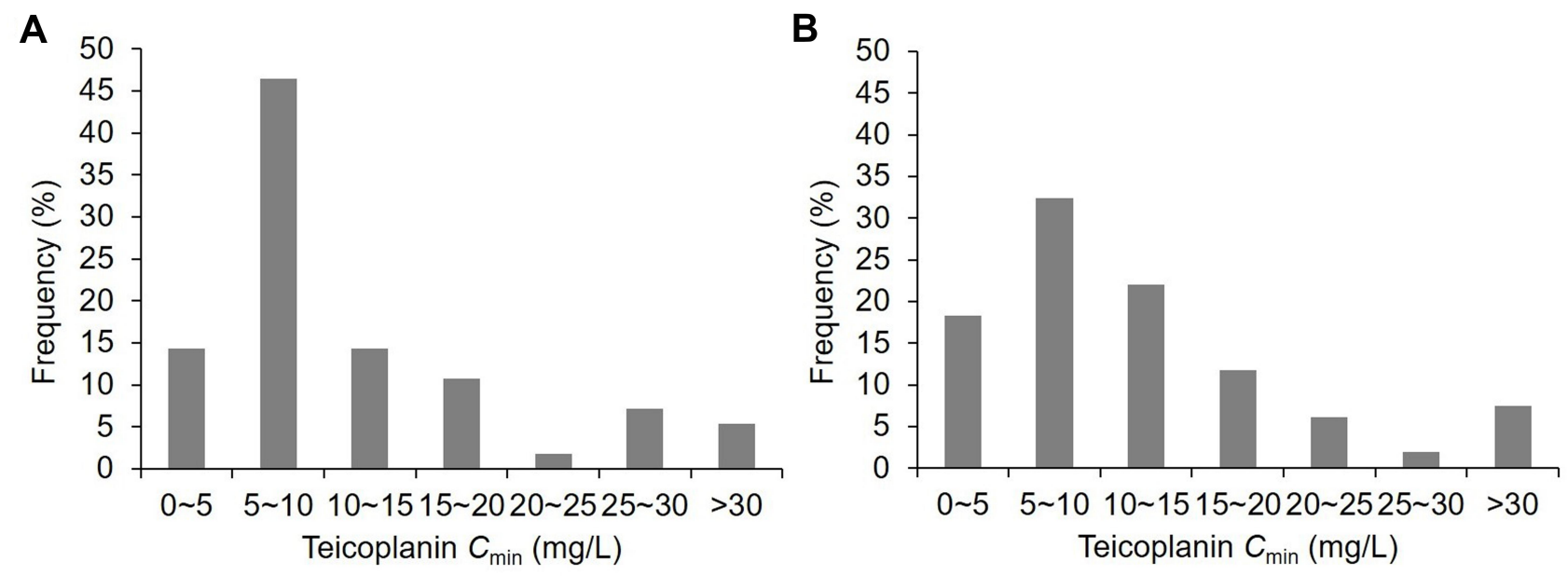

Figure I Distribution of teicoplanin trough concentrations. Panel $(\mathbf{A})$ shows the distribution of $C_{\min }$ on day 3 or day 4 after teicoplanin administration. Panel (B) shows the distribution of teicoplanin $C_{\min }$ at steady state.

and $52.7 \%$ of $C_{\min }$ is less than $10 \mathrm{mg} / \mathrm{L}$. Among the 269 teicoplanin $C_{\min }, 56 C_{\min }$ were obtained on day 3 or 4 after the initial teicoplanin administration, the median was $8.43 \mathrm{mg} / \mathrm{L}$ (IQR 6.41-15.02 mg/L) (Figure 1, panel A). The median of the remaining 213 teicoplanin $C_{\min }$ obtained at steady state was $9.77 \mathrm{mg} / \mathrm{L}$ (IQR 5.89-15.60 mg/L) (Figure 1, panel B). Compared with the steady state $C_{\min }$, those obtained on day 3 or day 4 were more likely to be $<10 \mathrm{mg} / \mathrm{L}$.

\section{Factors Influencing Teicoplanin C/D Factors Influencing Teicoplanin $\mathrm{C}^{3 / 4} \min / \mathrm{D}_{\mathrm{CLD}}$}

As shown in Table 2, gender, age, weight, concomitant use of carbapenems, and SCr were allowed to be included in the multivariate analyses due to those factors had the $P$ value $<0.1$. Finally, it was found that teicoplanin $C^{3 / 4} \min$ $/ \mathrm{D}_{\mathrm{CLD}}$ was significantly affected by age $(P=0.008)$, weight $(P=0.039)$, and $\operatorname{SCr}(P=0.022)$.

\section{Factors Influencing Teicoplanin $C_{\text {min }}^{S S} / D_{M D}$}

Accordingly, Table 3 shows the factors that influencing the teicoplanin $C^{\mathrm{SS}}{ }_{\min } / \mathrm{D}_{\mathrm{MD}}$. Although univariate analyses indicated that gender, weight, and $\mathrm{SCr}$ had the $P$ value $<0.1$, no significant correlation was found between weight and teicoplanin $C_{\text {min }}^{\mathrm{SS}} / \mathrm{D}_{\mathrm{MD}}$ in the multivariate analyses. Gender and $\mathrm{SCr}$ were found to be independently associated with teicoplanin $C_{\text {min }}^{\mathrm{SS}} / \mathrm{D}_{\mathrm{MD}}(P=0.014, P=0.006$, respectively $)$.

\section{Nephrotoxicity Analysis}

One hundred and eighty-two children were eligible for nephrotoxicity analysis. Nine of one hundred and eighty-two (4.9\%) patients occurred nephrotoxicity. As shown in Table 4, both univariable and multivariable logistic analysis indicated that teicoplanin $C_{\min }$ and the concomitant use of amphotericin B were the risk factors for the incidence of nephrotoxicity related to teicoplanin ( $P=0.012, P=0.001$, respectively). Further CART model showed that children with concomitant treatment with amphotericin B had teicoplanin $C_{\min }>9.81 \mathrm{mg} / \mathrm{L}$ or patients with teicoplanin $C_{\min }>21.94 \mathrm{mg} / \mathrm{L}$ were at high risk for nephrotoxicity (Figure 2). Inversely, patients who were concomitantly treated by amphotericin B with teicoplanin $C_{\min }<9.81 \mathrm{mg} / \mathrm{L}$ or those with teicoplanin $C_{\min }<21.94 \mathrm{mg} / \mathrm{L}$ were at low risk of nephrotoxicity.

\section{Discussion}

In this retrospective study, we described the distribution characteristics of teicoplanin $C_{\min }$ and investigated the potential factors affecting the concentration of teicoplanin based on 269 TDM data from 186 patients. In addition, we explored the risk factors related to the nephrotoxicity of teicoplanin and identified patients with high-risk of nephrotoxicity.

Teicoplanin $C_{\min }$ of $9.49 \mathrm{mg} / \mathrm{L}$ (IQR $5.97,15.35 \mathrm{mg} / \mathrm{L}$ ) was observed in this study. When $C_{\min }>10 \mathrm{mg} / \mathrm{L}$ was defined as the therapeutic target of teicoplanin, more than half of the teicoplanin $C_{\min }(52.7 \%)$ did not achieve the therapeutic target, which was similar to previous studies. ${ }^{16,26}$ Zhao et al reported the teicoplanin $C_{\text {min }}$ was $11.8 \mathrm{mg} / \mathrm{L}$ (IQR 3.0, $49.6 \mathrm{mg} / \mathrm{L}$ ), and $48 \%$ of children had sub-therapeutic steady-state $C_{\min }{ }^{16}$ Yamada et al reported 
Table 2 Univariate and Multivariate Analyses of the Variables Tested for Potential Association with $C^{3 / 4}{ }_{\min } / D_{C L D}$

\begin{tabular}{|c|c|c|c|c|}
\hline \multirow[t]{2}{*}{ Variables } & \multicolumn{2}{|c|}{$\begin{array}{l}\text { Univariate } \\
\text { Analysis }\end{array}$} & \multicolumn{2}{|c|}{ Multivariate Analysis } \\
\hline & $\mathbf{R}^{2}$ & $P$ value & $P$ value & $\beta(95 \% \mathrm{Cl})$ \\
\hline Gender (Male) & 0.082 & 0.032 & - & \\
\hline Age (year) & 0.209 & 0.001 & 0.008 & $0.69(0.019-0.119)$ \\
\hline Weight (kg) & 0.064 & 0.061 & 0.039 & $\begin{array}{l}-0.015 \\
(-0.029-0.001)\end{array}$ \\
\hline $\begin{array}{l}\text { Concomitant } \\
\text { medication }\end{array}$ & - & - & - & \\
\hline Antibacterial drugs & - & - & - & \\
\hline Cephalosporins & 0.023 & 0.270 & - & \\
\hline Carbapenems & 0.065 & 0.058 & 0.080 & $\begin{array}{l}-0.194 \\
(-0.413-0.024)\end{array}$ \\
\hline Antifungal drug & 0.001 & 0.856 & - & \\
\hline Antiviral drugs & 0.001 & 0.918 & - & \\
\hline Others & 0.035 & 0.138 & - & \\
\hline Comorbid conditions & - & - & - & \\
\hline Malignancy & 0.044 & 0.121 & - & \\
\hline $\begin{array}{l}\text { Congenital heart } \\
\text { disease }\end{array}$ & 0.002 & 0.771 & - & \\
\hline Hypoalbuminemia & 0.010 & 0.453 & - & \\
\hline WBC $\left(10^{9} / \mathrm{L}\right)$ & 0.004 & 0.636 & - & \\
\hline ALT (U/L) & 0.004 & 0.648 & - & \\
\hline AST (U/L) & 0.000 & 0.915 & - & \\
\hline Albumin $(g / L)$ & 0.023 & 0.268 & - & \\
\hline $\mathrm{SCr}(\mu \mathrm{mol} / \mathrm{L})$ & 0.279 & 0.001 & 0.022 & $\begin{array}{l}0.007 \\
(0.001-0.013)\end{array}$ \\
\hline $\operatorname{Ccr}(\mathrm{mL} / \mathrm{min})^{\mathrm{a}}$ & 0.048 & 0.106 & - & \\
\hline
\end{tabular}

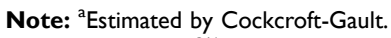

Abbreviations: $C^{3 / 4}{ }_{\min } / D_{C L D}$, the ratio between $C_{\min }$ on day 3 or day 4 after teicoplanin administration and the cumulative loading dose; $\mathrm{Cl}$ : confidence interval; WBC, white blood cell; ALT, alanine aminotransferase; AST, aspartate aminotransferase; $\mathrm{SCr}$, serum creatinine; $\mathrm{Ccr}$, creatinine clearance.

that the median $C_{\min }$ of teicoplanin was 16.3 (3.6, 25.9) $\mathrm{mg} / \mathrm{L}$ in children (measured by FPIA), and $46.2 \%$ of patients did not achieve a trough value $\geq 15 \mathrm{mg} / \mathrm{L}$ was in the recommended dose regimen group. ${ }^{17}$ Given those findings, it seems that teicoplanin $C_{\min }$ existed inadequate exposure in most patients. We suggest that the dose increase of teicoplanin and the exploration of $C_{\mathrm{min}^{-}}$ related influencing factors should be conducted in children to achieve better clinical responses.

In this study, age, weight, and $\mathrm{SCr}$ were found to be the factors that exerted a significant effect on teicoplanin $C^{3 / 4}{ }_{\min } / \mathrm{D}_{\mathrm{CLD}}$. Our results were partially similar with a previous study with a relatively small sample size (36 children) which reported that teicoplanin $C_{\min }$ would be influenced by age, $\mathrm{SCr}$ and estimated glomerular filtration when giving a fixed-dose. ${ }^{17}$ Since teicoplanin is
Table 3 Univariate and Multivariate Analyses of the Variables Tested for Potential Association with $C^{S S}{ }_{\text {min }} / D_{M D}$

\begin{tabular}{|l|l|l|l|l|}
\hline \multirow{2}{*}{ Variables } & \multicolumn{2}{l|}{$\begin{array}{l}\text { Univariate } \\
\text { Analysis }\end{array}$} & \multicolumn{2}{l|}{ Multivariate Analysis } \\
\cline { 2 - 5 } & $\mathbf{R}^{2}$ & $\mathbf{P}$ value & $\boldsymbol{P}$ value & $\boldsymbol{\beta}$ (95\% CI) \\
\hline Gender (Male) & 0.034 & 0.007 & 0.014 & 0.395 \\
Age (year) & & & & $(0.080-0.709)$ \\
Weight (kg) & 0.003 & 0.402 & - & \\
Concomitant medication & 0.025 & 0.021 & - & \\
Antibacterial drugs & - & - & - & \\
Penicillins & 0.004 & - & - & \\
Cephalosporins & 0.008 & 0.190 & - & \\
Carbapenems & 0.001 & 0.966 & - & \\
Antifungal drug & 0.002 & 0.512 & - & \\
Antiviral drugs & 0.001 & 0.650 & - & \\
Others & 0.012 & 0.118 & - & \\
Cumulative loading dose & 0.008 & 0.182 & - & \\
(mg/kg) & & & & \\
Comorbid conditions & - & - & - & \\
Malignancy & 0.010 & 0.153 & - & \\
Congenital heart & 0.012 & 0.115 & - & \\
disease & & & & \\
Hypoalbuminemia & 0.003 & 0.432 & - & \\
WBC (I09/L) & 0.001 & 0.727 & - & \\
ALT (U/L) & 0.001 & 0.965 & - & \\
AST (U/L) & 0.001 & 0.977 & - & \\
Albumin (g/L) & 0.001 & 0.891 & - & \\
SCr ( $\mu$ mol/L) & 0.041 & 0.003 & 0.006 & 0.014 \\
& & & & \\
Ccr (mL/min) & 0.004 & 0.352 & - & \\
\hline
\end{tabular}

Note: ${ }^{a}$ Estimated by Cockcroft-Gault.

Abbreviations: $C^{S S}{ }_{\min } / D_{M D}$, the ratio between the teicoplanin $C_{\min }$ at steady state and the maintenance dose; Cl: confidence interval; WBC, white blood cell; ALT, alanine aminotransferase; AST, aspartate aminotransferase; $\mathrm{SCr}$, serum creatinine; Ccr, creatinine clearance.

mainly excreted by the kidneys, ${ }^{27}$ impaired renal function usually causes a decrease of teicoplanin clearance. ${ }^{28}$ In addition, drug clearance and volume of distribution in children also changed with the increase of age and weight, further affecting the drug exposure. Strenger et al found that compared with school-age children, young children had significantly lower initial teicoplanin $C_{\text {min. }}{ }^{19}$ Besides, several studies have also indicated that weight could significantly influence the pharmacokinetics of teicoplanin in children. ${ }^{3,16,29}$ Therefore, it is necessary to consider age, weight and serum creatinine to achieve the therapeutic target of teicoplanin in children as soon as possible.

For the $C_{\text {min }}^{\mathrm{SS}} / \mathrm{D}_{\mathrm{MD}}$, in addition to $\mathrm{SCr}$, gender was also found to be significantly related to teicoplanin $C^{\mathrm{SS}}{ }_{\min } / \mathrm{D}_{\mathrm{MD}}$. Similar to the recent results concluded 
Table 4 Univariable and Multivariable Analysis of Risk Factors for Nephrotoxicity in Patients Receiving Teicoplanin

\begin{tabular}{|l|l|l|l|l|}
\hline \multirow{2}{*}{ Variables } & \multicolumn{2}{|l|}{$\begin{array}{l}\text { Univariate } \\
\text { Analysis }\end{array}$} & \multicolumn{2}{l|}{ Multivariate Analysis } \\
\cline { 2 - 5 } & $\begin{array}{l}\text { Wald } \\
\chi^{2}\end{array}$ & $P$ value & P value & OR (95\% CI) \\
\hline Gender (Male) & 0.816 & 0.217 & - & \\
Age (year) & 0.003 & 0.956 & - & \\
Weight (kg) & 0.013 & 0.908 & - & \\
Concomitant & - & - & - & \\
nephrotoxic drugs & & & & \\
Aminoglycosides & 0.001 & 0.999 & - & \\
Loop diuretics & 1.731 & 0.188 & - & \\
Acyclovir & 0.001 & 0.999 & - & \\
Amphotericin B & 15.509 & 0.001 & 0.001 & 0.285 \\
Cis-platinum & 0.001 & 0.999 & - & $(0.183-0.388)$ \\
Comorbid conditions & - & - & - & \\
Malignancy & 0.373 & 0.541 & - & \\
Congenital heart & 1.005 & 0.316 & - & \\
disease & & & & \\
Hypoalbuminemia & 0.179 & 0.672 & - & \\
Teicoplanin $C_{\text {min }}(\mathrm{mg} / \mathrm{L})$ & 5.511 & 0.019 & 0.012 & 0.005 \\
Baseline SCr ( $\mu$ mol/L) & 0.078 & 0.780 & - & $(0.001-0.008)$ \\
Baseline Ccr (mL/min) & 0.034 & 0.853 & - & \\
\hline
\end{tabular}

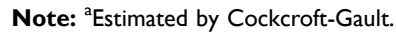

Abbreviations: $\mathrm{OR}$, odds ratio; $\mathrm{Cl}$ : confidence interval; $C_{\text {min, }}$, trough concentration; $\mathrm{SCr}$, serum creatinine; $\mathrm{Ccr}$, creatinine clearance.

from Cai et al, which demonstrated that there were gender-related differences in teicoplanin $C_{\min }{ }^{29}$ Another study found that adolescent girls had significantly higher initial and follow-up teicoplanin $C_{\min }$ than adolescent boys, and the authors suggested that genderrelated differences in protein binding, tissue distribution or renal excretion of teicoplanin may explain this difference. ${ }^{19}$ More prospective studies are expected in the future to explore the causes of this phenomenon.

In the field of TDM, studies rarely focused on the nephrotoxicity of teicoplanin in children. The incidence of nephrotoxicity was $4.9 \%$ according to the current study. A retrospective study found that the overall incidence of nephrotoxicity in children was $2.3 \%{ }^{17}$ We found that teicoplanin $C_{\min }$ and concomitant treatment with amphotericin B were independent risk factors for nephrotoxicity of teicoplanin in children. Moreover, there was an increased risk of nephrotoxicity when the $C_{\min }$ of teicoplanin exceed $21.94 \mathrm{mg} / \mathrm{L}$ in children or the $C_{\min }$ exceed $9.81 \mathrm{mg} / \mathrm{L}$ in children with concomitant use of amphotericin B. To our knowledge, this is the first study to report the risk factors of teicoplanin-related nephrotoxicity and the high-risk population in children. Previous studies reported that a $C_{\min }$ of $>60 \mathrm{mg} / \mathrm{L}$ increased the likelihood of nephrotoxicity of teicoplanin. ${ }^{6}$ Similarly, Wilson reported that adult patients with teicoplanin $C_{\min }>$ $60 \mathrm{mg} / \mathrm{L}$ had a higher incidence of nephrotoxicity compared to patients with teicoplanin $C_{\min }$ of $20-40 \mathrm{mg} / \mathrm{L}{ }^{30}$ However, another study drew a contradictory conclusion that there was no significant correlation between the $C_{\min }$ and the occurrence of nephrotoxicity in adults. ${ }^{5}$ The most common adverse reaction of amphotericin B is nephrotoxicity. A previous study showed that amphotericin B combined with vancomycin was associated with impaired renal function. ${ }^{31}$ In this study, 4 of 9 patients developing nephrotoxicity were received concomitant treatment with amphotericin B. The nephrotoxicity threshold of teicoplanin was decreased from $21.94 \mathrm{mg} / \mathrm{L}$ to $9.81 \mathrm{mg} / \mathrm{L}$ when combining amphotericin B and teicoplanin, it may be caused by the synergy between the two drugs. This result suggested that the concomitant use of amphotericin B could aggravate the nephrotoxicity possibility of teicoplanin. Therefore, more attention should be paid to the children who received concomitant treatment with amphotericin B and teicoplanin in the future, even when the $C_{\min }$ does not reach the therapeutic target.

There are several limitations in our study. Firstly, this study was a retrospective study, neither the number of TDM samples nor the timing of TDM for each patient was consistent. Secondly, the relationship between drug exposure and clinical efficacy was not explored because there are few microbiologically documented gram-positive bacterial infections in this study. Larger-scale, multicenter and prospective studies are expected to further validate the results of teicoplanin TDM in children.

\section{Conclusion}

This is a retrospective study of teicoplanin in a larger sample size of children patients. The current dose regimen of teicoplanin may result in inadequate drug exposure in children. Age, weight, gender and $\mathrm{SCr}$ are important factors that can significantly influence the fluctuation of teicoplanin $C_{\min }$. Teicoplanin $C_{\min }$ and concomitant treatment with amphotericin $\mathrm{B}$ were independent risk factors for nephrotoxicity. It is recommended to perform TDM in children to ensure the efficacy and safety of teicoplanin. 


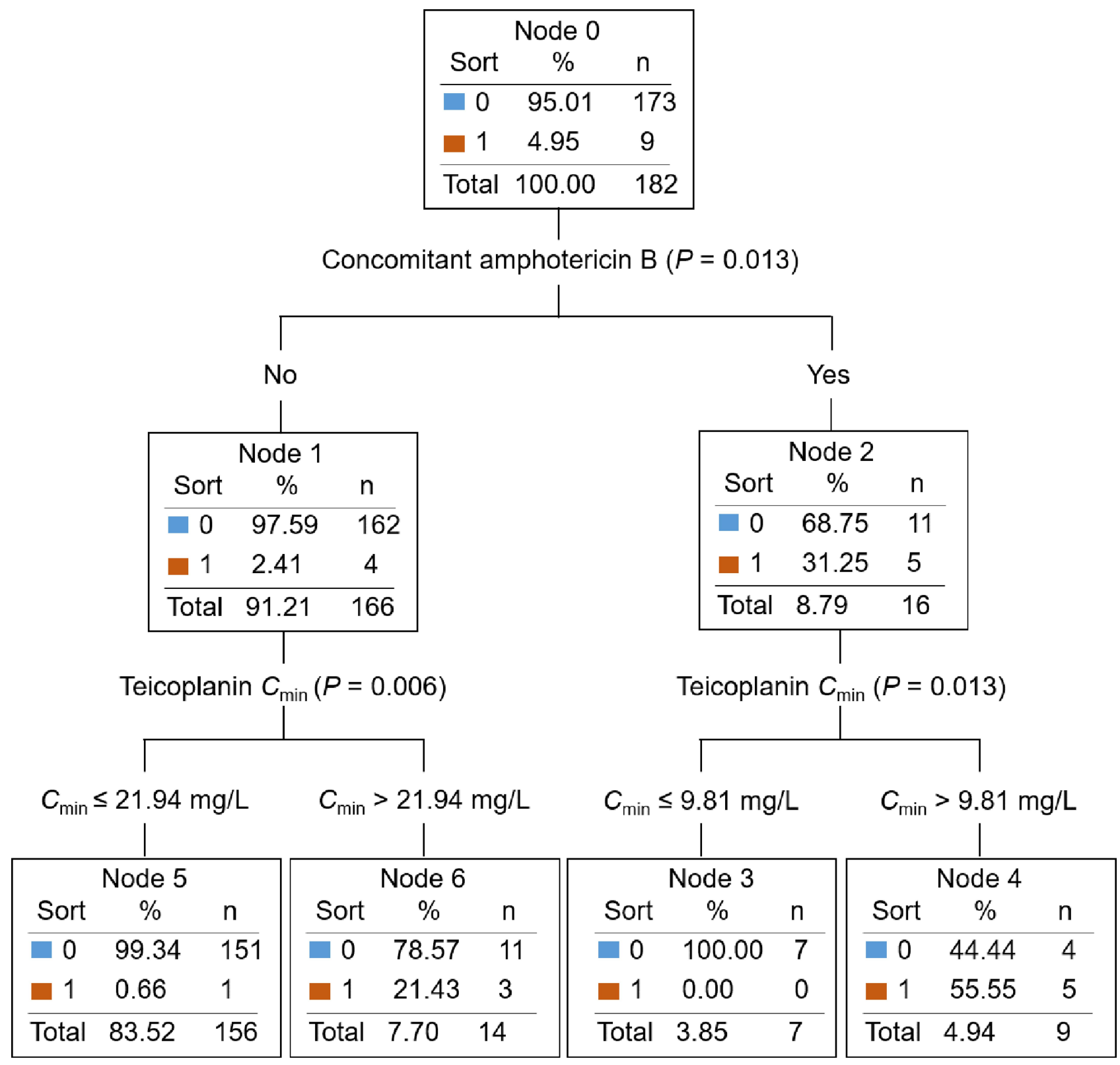

Figure 2 Classification and regression tree model for the nephrotoxicity of teicoplanin therapy. A value of 0 indicated that no nephrotoxicity was observed and a value of $\mathrm{I}$ indicated that nephrotoxicity was observed.

\section{Ethics Approval and Informed Consent}

This study was approved by the Ethics Committee of the First Affiliated Hospital of Xi'an Jiaotong University and the Ethics Committee of Xi'an Children's Hospital, respectively. Waiving of informed consent was given due to the retrospective, non-interventional study design. All patient data were collected anonymously. This study was performed in accordance with the Declaration of Helsinki. Patients were ensured about the confidentiality of their information.

\section{Author Contributions}

All authors made a significant contribution to the work reported, whether that is in the conception, study design, execution, acquisition of data, analysis and interpretation, or in all these areas; took part in drafting, revising or critically reviewing the article; gave final approval of the version to be published; have agreed on the journal to which the article has been submitted; and agree to be accountable for all aspects of the work. 


\section{Funding}

This study was supported by the Special Fund for Drug Development and Rational Application of Chinese Pharmacological Society (No. CTDM (XM-2017-01-00114)) and the Key Research and Development Program in Shaanxi Province of China (No. 2019ZDLSF01-05) for supporting this study.

\section{Disclosure}

Dan Sun and Tao Zhang are co-first authors for this study. The authors declare that they have no conflicts of interest.

\section{References}

1. Venkatesh MP, Placencia F, Weisman LE. Coagulase-negative staphylococcal infections in the neonate and child: an update. Semin Pediatr Infect Dis. 2006;17(3):120-127. doi:10.1053/j.spid.2006. 06.005

2. Maldonado ME, Acuña M, Álvarez AM, et al. Microorganismos aislados de hemocultivos en niños con cáncer y neutropenia febril de alto riesgo en cinco hospitales de Santiago, Chile, período 20122015. Revista Chilena de Infectología. 2018;35(2):140-146. doi: $10.4067 / \mathrm{s} 0716-10182018000200140$

3. Ramos-Martin V, Paulus S, Siner S, et al. Population pharmacokinetics of teicoplanin in children. Antimicrob Agents Chemother. 2014;58(11):6920-6927. doi:10.1128/AAC.03685-14

4. Spencer CM, Bryson HM. Teicoplanin. A pharmacoeconomic evaluation of its use in the treatment of gram-positive infections. Pharmacoeconomics. 1995;7(4):357-374. doi:10.2165/00019053199507040-00009

5. Zhou LJ, Gao YQ, Cao W, et al. Retrospective analysis of relationships among the dose regimen, trough concentration, efficacy, and safety of teicoplanin in Chinese patients with moderate-severe Grampositive infections. Infect Drug Resist. 2018;11:29-36. doi:10.2147/ IDR.S146961

6. Abdul-Aziz MH, Alffenaar JC, Bassetti M, et al. Antimicrobial therapeutic drug monitoring in critically ill adult patients: a position paper. Intensive Care Med. 2020;46:1127-1153.

7. Wang TT, Li N, Hu SS, et al. Factors on trough teicoplanin levels, associations between levels, efficacy and safety in patients with gram-positive infections. Int J Clin Pharmacol Ther. 2015;53 (05):356-362. doi: $10.5414 / \mathrm{CP} 202247$

8. Targocid EMC 200mg powder for solution for injection/infusion or oral solution. Available from: https://www.medicines.org.uk/emc/pro duct/2926/smpc. Accessed 29 June 2020.

9. Yuan DX. Teicoplanin for infusion. Available from: http://drugs.dxy. $\mathrm{cn} / \mathrm{drug} / 82481 /$ detail.htm\#4. Accessed 29 June 2020.

10. Kim S-H, Kang C-I, Huh K, et al. Evaluating the optimal dose of teicoplanin with therapeutic drug monitoring: not too high for adverse event, not too low for treatment efficacy. Eur J Clin Microbiol Infect Dis. 2019;38(11):2113-2120. doi:10.1007/s10096019-03652-6

11. Barco S, Mesini A, Barbagallo L, et al. A liquid chromatography-tandem mass spectrometry platform for the routine therapeutic drug monitoring of 14 antibiotics: application to critically ill pediatric patients. J Pharm Biomed Anal. 2020;186:113273. doi:10.1016/j.jpba.2020.113273

12. Yoshida T, Yoshida S, Okada H, et al. Risk factors for decreased teicoplanin trough concentrations during initial dosing in critically ill patients. Pharmazie. 2019;74:120-124.
13. Kato H, Hamada Y, Hagihara M, et al. Retrospective study of teicoplanin loading regimen that rapidly achieves target $15-30 \mu \mathrm{g} / \mathrm{mL}$ serum trough concentration. $J$ Infect Chemother. 2016;22 (5):308-313. doi:10.1016/j.jiac.2016.01.019

14. Byrne CJ, Egan S, Fennell JP, et al. Teicoplanin use in adult patients with haematological malignancy: exploring relationships between dose, trough concentrations, efficacy and nephrotoxicity. Int J Antimicrob Agents. 2015;46(4):406-412. doi:10.1016/j.ijantimicag.2015.05.019

15. Ramos-Martin V, Neely MN, McGowan P, et al. Population pharmacokinetics and pharmacodynamics of teicoplanin in neonates: making better use of C-reactive protein to deliver individualized therapy. J Antimicrob Chemother. 2016;71(11):3168-3178. doi:10.1093/jac/dkw295

16. Zhao W, Zhang D, Storme T, et al. Population pharmacokinetics and dosing optimization of teicoplanin in children with malignant haematological disease. Br J Clin Pharmacol. 2015;80(5):1197-1207. doi:10.1111/bcp. 12710

17. Yamada T, Kubota T, Yonezawa M, et al. Evaluation of teicoplanin trough values after the recommended loading dose in children with associated safety analysis. Pediatr Infect Dis J. 2017;36(4):398-400. doi:10.1097/INF.0000000000001456

18. Yamada T, Kubota T, Nakamura M, et al. Evaluation of teicoplanin concentrations and safety analysis in neonates. Int J Antimicrob Agents. 2014;44(5):458-462. doi:10.1016/j.ijantimicag.2014.07.005

19. Strenger V, Hofer N, Rödl S, et al. Age- and gender-related differences in teicoplanin levels in paediatric patients. J Antimicrob Chemother. 2013;68:2318-2323.

20. Cavalcanti AB, Goncalves AR, Almeida CS, et al. Teicoplanin versus vancomycin for proven or suspected infection. Cochrane Database Syst Rev. 2010;6:CD007022.

21. Kato-Hayashi H, Niwa T, Ohata K, et al. Comparative efficacy and safety of vancomycin versus teicoplanin in febrile neutropenic patients receiving hematopoietic stem cell transplantation. J Clin Pharm Ther. 2019;44(6):888-894. doi:10.1111/jcpt.13011

22. Moffett BS, Goldstein SL. Acute Kidney Injury and Increasing Nephrotoxic-Medication Exposure in Noncritically-Ill Children. Clin J Am Soc Nephrol. 2011;6(4):856-863. doi:10.2215/CJN.0811 0910

23. Hu S, Pang C, Dong H, et al. Determination of teicoplanin serum concentration in critically ill patients. Chine J Hospital Pharmacy. 2011;31:753-755.

24. Sánchez A, López-Herce J, Cueto E, et al. Teicoplanin pharmacokinetics in critically ill paediatric patients. $J$ Antimicrob Chemother. 1999;44(3):407-409. doi:10.1093/jac/44.3.407

25. Kellum JA, Bellomo R, Ronco C. Definition and classification of acute kidney injury. Nephron Clin Pract. 2008;109(4):c182-187. doi: $10.1159 / 000142926$

26. Jung J, Lee K, Oh J, et al. Therapeutic drug monitoring of teicoplanin using an LC-MS/MS method: analysis of 421 measurements in a naturalistic clinical setting. $J$ Pharm Biomed Anal. 2019;167:161-165. doi:10.1016/j.jpba.2019.02.001

27. Bernareggi A, Borghi A, Borgonovi M, et al. Teicoplanin metabolism in humans.. Antimicrob Agents Chemother. 1992;36(8):1744. doi:10.1128/AAC.36.8.1744

28. Gao LL, Xu H, Ye Q, et al. Population pharmacokinetics and dosage optimization of teicoplanin in children with different renal functions. Front Pharmacol. 2020;11:552. doi:10.3389/fphar.2020.00552

29. Cai J, Liu XF, Ni HQ. Analysis of monitoring results and influencing factors of blood drug concentration of different dosages of teicoplanin. Pharmacy Today. 2020;30:565-568.

30. Wilson AP. Comparative safety of teicoplanin and vancomycin. Int J Antimicrob Agents. 1998;10(2):143-152. doi:10.1016/S09248579(98)00025-9

31. Feiten HDS, Okumura LM, Martinbiancho JK, et al. Vancomycinassociated nephrotoxicity and risk factors in critically ill children without preexisting renal injury. Pediatr Infect Dis J. 2019;38 (9):934-938. doi:10.1097/INF.0000000000002391 


\section{Publish your work in this journal}

Infection and Drug Resistance is an international, peer-reviewed openaccess journal that focuses on the optimal treatment of infection (bacterial, fungal and viral) and the development and institution of preventive strategies to minimize the development and spread of resistance. The journal is specifically concerned with the epidemiology of

Submit your manuscript here: https://www.dovepress.com/infection-and-drug-resistance-journa| antibiotic resistance and the mechanisms of resistance development and diffusion in both hospitals and the community. The manuscript management system is completely online and includes a very quick and fair peerreview system, which is all easy to use. Visit http://www.dovepress.com/ testimonials.php to read real quotes from published authors. 